\title{
Effects of Player's Participation Patterns on Economic Performance of
}

\section{Entertainment Shopping Website}

\author{
Jin $\mathrm{Li}^{1{ }^{1,},}$ Kwok Fai Tso ${ }^{2}$ \\ ${ }^{1}$ Xidian University, Xi'an, Shaanxi, China \\ ${ }^{2}$ City University of Hong Kong, Hong Kong \\ 1 jinli@xidian.edu.cn*; ${ }^{2}$ msgtso@ cityu.edu.hk; \\ * corresponding author
}

\begin{abstract}
Online pay-per-bid auction is a new entertainment shoppingformat with a mechanism that integrates the characteristics of traditional e-commerce, online auctions, lotteries and games. Previous researches have shown this auction format possesses high variances and uncertainties. However, research on the players' participation patterns and performance is rare. In this study, we attempt to study how players' participation patterns can affect both their own and websites' performance. An empirical study with data collected from an online pay-per-development website with 5,650players' participation behavior is performed. Particularly, the role of players preference is also explored through a cluster analysis of their historical participations. Multiple linear regression models and regression models with beta distributions are used for hypothesis testing. Findings confirm that players participation performance and websites revenue strongly associated with the players participation patterns. The results show that (1) players with longer lifetime perform better and contribute more revenue to the website; (2) players participating in more auctions show worse performance and websites obtain more revenue from them; and (3) players with a strong preference behave more irrationally and website benefits more from them. Both theoretical and managerial implications are discussed.
\end{abstract}

Keywords: Online Entertainment Shopping; Pay-per-bid Auctions; Player Learning; Risk Attitude; Bidding Performance; Website Profit;

\section{Introduction}

Most online entertainment shopping websites adopt the pay-per-bid auction mechanism as the bidding rule [16]. The website sets an auction with a starting price at $\$ 0$. Players bid to express their purchase intentions and a countdown clock is attached to raise the nervousness of the bidders [15]. Players pay a fixed fee for each bid, e.g., $\$ 1$, and the ending price of the auction is increased by only a small increment, e.g., $\$ 0.01$. For typical online auctions like the ones held on eBay, the auction is over when the clock counts down to the end. For entertainment shopping, the clock works till the remaining time is less than the "roll-back" period, e.g., 10 seconds. Once a new bid comes in, the clock is extended by 10 seconds again, that's why it's called the "roll-back" period. The auction ends when no new bid comes in and the last player is the final winner. The website gets its revenue from players' devoted bidding fees plus the final ending price paid by the winner. The cost is mainly the cost of purchasing of products, shipments and website operations. From the players' point of view, the winner buys the product at a large discount. The cost involves only the bidding fee and the final price of purchasing the product, which is usually much lower than the product's retail price [19]. On the other hand, losers lose all sunk costs and only enjoy their participation. In this way, the website packages itself as e-commerce with characteristics of both entertainment and pay-per-bid auction [17].

Previous researches showed that this auction format has high variances and uncertainties [32]. These researchers paid more attention to the mechanism details of pay-per-production and the theoretical predictions of ending prices [16, $19,32]$, but neglected the specialties that exist in conventional electronic markets. We wonder if these traditional characteristics (such as registered users' adherence to the website [9], their learning through experience [34], their attitudes towards risk [12], their product taste or preference [29] and so on) perform the same in online entertainment shopping websites. We aim to find these effects in online pay-per-bid auctions and answer the following questions: How do players with different lifetimes contribute revenue to the website? Which group of players contributes the most profit to the website? Do players have specific auction type preferences? Which characteristics can help players to make more effective biddings? How are players' behaviors differ if they have different auction type preferences? 
To investigate these effects, several factors from the conventional e-commerce and traditional online auction, such as player adherence, learning, preference, and risk attitude, were examined through hypothesis testing. More details for these factors and hypotheses are described in Section2. The rest of this paper is organized as follows. In Section 3, the research context and descriptive statistics for the auctions provided by the website are presented. Section 4 introduces the sample players used for hypothesis testing and describes the variables defined for empirical tests. Cluster analysis is also employed to classify players into different preference groups, according to their historical participations. In Section 5, we use linear regression model and regression model with beta distribution to empirically test the types introduced in Section 2. Discussions and research limitations are provided in Section 6.

\section{Hypothesis}

\subsection{Player Adherence}

According to the mechanism of pay-per-production websites, keeping a crowd of online players for competition is the core part in generating considerable revenue. No bidding competition would lead players to win with a very low price and a small amount of sunk cost. Players involved in the game consist of those who are newly registered and those who have previously participated in many auctions. Keeping players adhering to the website can reduce the cost of acquiring new ones [9]. What methods can be used by the websites to improve the players' adherence? For the success of internet firms, consumer loyalty acts as a critical determinant [3]. Online consumers' high level of loyalty is the motivation factor for them to participate repeatedly in online games [5]. Moreover, consumers 'loyalty is used to measure their tendency to repeatedly purchase the products or services from the same firm [13,24]. To such online firms, methods to include customers' loyalty are needed. Online community or game is a widely used business model by electronic markets to increase users' loyalty [4]. Such kind of online community or game can bring entertainment to users [8]. Moreover, the rewards associated with engaging can also be a motivation for being active [25]. Pay-per-auction websites also create such online games to keep its players by rewarding them. The websites encourage players to log in to the website and play the provided free games. Some bidding tokens for free auction games would be allocated to them for being active online. We define player adherence as the degree of players' levels of activities, measured by both active lifetime and auction participations. Furthermore, we hypothesize that:

H1. Players with a longer lifetime contribute more profit to the entertainment shopping website.

H2. Players who participate in auctions more frequently contribute more profit to the entertainment shopping website.

\subsection{Player Learning}

Uncertainties are associated with new products or services when they are introduced to consumers who need to undergo a period called "learning" process [28] to reduce these uncertainties. In marketing, uncertainties always refer to qualities of new products or services, e.g., laundry detergent [30], TV brand choices [31], and new pharmaceutical medicine [28]. Iyengar et al. [24] apply the concept of learning to the mobile market as users are uncertain about their data usage and the company's wireless service quality before they buy a plan. Recently, consumer learning is also studied in many information system researches. Mobile users may need the learning process to understand the mobile activities they are truly interested in [1]. Consumers can learn from online product reviews to make better purchasing decisions [35]. Online users need to learn their potential for creative ideas through peer votes on crowdsourcing websites [34]. Does this learning process exist in online pay-per-bid auctions? Competition is one of the characters of online auctions. Based on previous literature, persistent players on entertainment shopping websites may learn to perform better in the bidding game. We use the proportion of no loss biddings, which is also treated as the proportion of effective biddings, to measure players' bidding performance. A higher proportion means a better bidding performance, whereas "no loss" means that players have no monetary losses in biddings. More details will be elaborated in Section 4.Accordingly, we shall test the following hypotheses:

H3. Players with a longer lifetime make a higher proportion of effective biddings.

H4. Players who participate in auctions more frequently make a higher proportion of effective biddings.

\subsection{Risk Attitudes}

Consumers face many uncertainties with a virtually remote online seller but not with a real retailer [12]. Hence, trust is an important factor in the e-commerce market [6]. No online community or social network for entertainment 
shopping websites is set up in case of cooperation among online bidders, which brings the problem of information asymmetry: what should be done to make users believe in the reality of the auction website as they need to pay to bid with monetary risks? Online sellers apply different ways to show their quality signals to accent consumers' trust [27], including direct quality, indirect quality, and seller credibility indicators. The warranting principle [14] states that online consumers are more likely to trust signals when personal information is difficult or costly to feign, such as word of mouth [10]. Sellers are advised to encourage user-generated contents [33], considering their advantages. Accordingly, the pay-per-production website opens a platform for its users to post their real pictures along with the product they won to increase the website's credibility and consumers' trust. From a firm's view, it can increase the website's credibility to attract more new consumers and provide a communication channel between players and the website. From a consumer's perspective, these pictures can reduce the risk associated with fake selling [20] and help devote money into participations. Players who pay more attention to paid pay-per-auction are seen as players with a risk-seeking attitude. Moreover, they show different levels of bidding aggressions, reflected by the sunk cost they expect to devote in each auction. Considering these risk attitudes, the following hypothesis is presented:

H5. Players with a risk-averse attitude make a higher proportion of effective biddings.

\subsection{Player Reference}

As online pay-per-production combines both entertainment games and auctions, strategies used in electronic markets can be borrowed into this new market. Players participate in online games mainly for entertainment and fun [4], which proves that their preference is positively related with their loyalty. Hsu and $\mathrm{Lu}$ [4] define preference as the degree of players' positive feelings when participating in online games. Players Would like to devote their time into such games if they can obtain positive feelings or just enjoy it [29]. Auction fever is often used to understand the feelings in auctions [11]. Players may irrationally bid much more than their expectations. Considering the psychological influence in auctions, Adam et al. [18] develop a framework for emotional bidding and conclude that "electronic markets for consumers have recently begun to compete in 'emotions', rather than focusing solely on simple economics and price wars". They incorporate the preference factor into their emotional bidding framework but without empirical evidence. Normally, entertainment shopping websites set up many types of auctioned products to attract players with various tastes. Recently, some online entertainment shopping websites that aim to attract players with specific common interests have already broken out. RoyalKingBids.com and StarBid.com are two websites that target players interested in cigars, while PennyGunAuctions.com and BidGunner.com are for players who like shooting sports. Because the websites have their own classification types for auctioned products, each of these types has its common attributes. We will not get involved with causes of players' preference. Players may prefer one type of auction as the products belonging to that type can provide them a higher match value [1]. Alternatively, they perceive themselves to have a higher winning probability for that auction type [18]. Accordingly, we propose the following hypotheses:

H6. Players with a strong auction type preference contribute more profit to the entertainment shopping website.

H7. Players with a strong auction type preference with a larger monetary risk make a lower proportion of effective biddings.

\section{Research Context}

We collected data from a leading entertainment shopping website in China. The website sells a large number of consumer electronics and other popular products sold in typical online retail stores. Regarding the online entertainment shopping website we focused on, any individual with a cell phone number can register to that website and participate in the ongoing auctions provided there. The website provides two categories of bidding tokens: free bidding tokens (FBT) for risk-averse players and paid bidding tokens (PBT) for risk-seeking players. Players can be rewarded with few FBTs after a successful registration. Then, they have to either buy PBT (USD0.16 for each) or take part in activities held by the website (e.g., online games) to earn FBT for more participation. FBT can only be used in free auctions, but PBT is applicable for both free and paid auctions. However, in reality, nobody would like to devote PBT into free auctions.

During the data collection period, the website provided a total of 1,274 different products and categorized them into 5 types of auctions, wherein the first 4 belonging to paid auctions: Virtual Products (VP, eg, phone top-up cards, and 
paid bidding tokens. ), General Merchandises (GM, eg, outdoor gears and kitchen supplies), Cheap Digital Products (CDP, eg, computer accessories, such as mice and earphones), Expensive Digital Products (EDP, eg, cellphones and laptops), and Free Auctions (FA, eg, flash disks and digital albums). Except for the value difference of auctioned products between CDP and EDP, most auctions belonging to EDP require more than 1 (around 2 or 3) PBT for each bid. Correspondingly, the ending price would also be increased with the same multiple, around 2 or 3 cents rather than one cent. Hence, compared to other auction types, EDP involves more risks and uncertainties. During the data collection period, 21,463 completed auctions covering 650 products were observed. From the view of leverage profit, the website incurred loss for GM and FA but benefited from VP, CDP, and EDP. Although there were very few EDP auctions, as they represent less than $3 \%$ of all auctions, they contribute the most profit per auction.

Players could view the username of the current leader (some players used aggressive or tricky names to express aggressive signals or other special meanings, eg, "I am new", "thanks for giving it to me", "I will bid to the end ", And so on) and the remaining time for an ongoing auction. For the ended auctions, historical auction closing prices and winner information, i.e. username, id, and winning history, were disclosed to all individuals, so players could observe the information so they can make wiser decisions.

\section{Sample and Measures}

\subsection{Sample}

We tracked players bidding behavior in live auctions for more than three months from September 26, 2011 to January21, 2012. During this period, the website provided a relatively stable number of auctions and attracted a steady stream of newly registered players every day. registered during the observation period are chosen to do the analysis. As players may be uncertain about their auction type choices and participate in auctions randomly at the registration beginning. We need to observe their auction participation behaviors for a relatively long time to make sure their participation has reached the steady state. For consistency in the analysis, only 10,428 players who have registered during the first 30 days are selected. These players were all new members to the websites. Moreover, those who registered in the first month could have enough time, around two months, to experience different auction types and make decisions on which activities to participate in. Among these players, 4,778 members participated in free auctions only. These bidders were eliminated as they did not convert from Free Member group to Paid Member group, which means that no positive monetary revenue was produced by these users. Thus, only the remaining 5,650 members were included in our analysis.

\subsection{Measures}

As we hypothesized the effects of players 'behavior on entertainment shopping website antecedently, variables related to players' attributes and historical participation behaviors were extracted from the dataset. Table 1 summarizes the variables collected from the data.

Web The profile is used to capture the monetary profit that the website obtained from the player. It is calculated by summing up the bidding fee (for which the PBT is a proxy) and closing price paid by a player minus the retail price of the auctioned product.

PropNoLossBidfor each player is used to measure the player 'effective or rational bidding levels, defined as the proportion of bids where the player did not lose any money. For a player, it is a fraction value from 0 to 1 calculated by the summation of bids he / she placed in auctions won by him / her plus the number of his / her free bids in the losing auctions, divided by the number of all bids placed by this player. 
Jin Li \& Kwok Fai Tso / Vol. 2, No. 3, December 2019, pp. 102-112

Table. 1. Variable Definitions

\begin{tabular}{|l|l|}
\hline Variable Name & Definition \\
\hline WebProfit & Website profit from a specific player \\
\hline PropNoLossBid & $\begin{array}{l}\text { Proportion of bids which are placed without loss, i.e. free bids or paid } \\
\text { bid for auctions won by the player }\end{array}$ \\
\hline TrickyName & If a tricky name is used \\
\hline RegTime & Player's registration time (day) \\
\hline TimeGap & Days between registration and first bid \\
\hline Duration & Days between the first and last bids during the time period \\
\hline AvgPaidAuction & Average number of paid auctions participated per day \\
\hline AvgFreeAuction & Average number of free auctions participated per day \\
\hline AvgPaidBid & Average number of paid bidding in paid auctions \\
\hline AvgFreeBid & Average number of free biddings in free auctions \\
\hline WinProb & Winning probability for auctions including both paid and free ones \\
\hline PreferenceType & Which auction type preference cluster the player belongs to \\
\hline
\end{tabular}

TrickyName, RegTime, and TimeGapare introduced as control variables for individual effects. TrickyNameis used to check whether the player used a tricky name that transferred aggressive information or not. RagTime is used to capture the time point of registration during the registration period. TimeGapis used to find out how quickly the player began to participate in auctions after the registration.

Duration is for the player's lifetime, measured by days from the first to the last time of bidding, and used to measure the players' adherence to the website in time dimension.

AvgPaidAuctionand AvgFreeAuctionare used to measure players' adherence in auction participation dimension. They are calculated by the total number of paid auctions (NumPad Auction) or total number of free auctions (NumFreeAuction) participated in, divided by their corresponding durations.

AvePaidBidand AveFreeBidare used for players' aggressive leveling bidding dimensions for paid and free auctions respectively. The larger value indicates the higher level of player aggressiveness in the paid and free auctions.

WinPro measures the player's winning probability, calculated by the number of winning auctions divided by the number of all auctions participated in.

Players are heterogeneous as every individual has their own product taste or preference. For registered players, some of them participated only for entertainment and enjoyed leisure on the internet, especially those who only joined the free auctions. Some others devoted themselves into gambling, aiming to win profit and enjoyed the excitement of winning. This attitude determines players bidding preferences and participation patterns. As different players have participated in various total numbers of auctions, we transformed the number for each type into a percentage of this type's auction number, relative to the bidder's total auction number. For example, if a player participated in 100 auctions in total, and among which were $60 \mathrm{VP}$ auctions, $20 \mathrm{GM}$ auctions, $10 \mathrm{CDP}$ auctions, 5 EDP auctions and 5 free auctions. The transformed percentages of each type's auction number are 0.6, 0.2, 0.1, 0.05 and 0.05 respectively.

Cluster analysis can be used to segment players according to their historical participation data. The k-means clustering method was used to allocate all players into k clusters, in which each cluster has the nearest distance to the centroid. Analysis to minimize the within-cluster sum of squares (WCSS) for all clusters applied. We iteratively apply the k-means method 100 times for each k, from 2 to 20, to calculate the average WCSS for each $\mathrm{k}$.

We need to identify the best $\mathrm{k}$ values for our dataset. Similarly, with the iterations used for WCSS, we calculated the criteria values to identify the number of clusters based on three criteria: Calinski-Harabasz pseudo F-statistic (CHPF) [22], Davies-Bouldin index (DBI) [7], and silhouette value (SV) [21]. CHPF is used to measure the separation between clusters, and a higher statistic value indicates better separation. Average SV for the entire dataset is used to measure how appropriately the data has been clustered, and the value close to 1 is preferred for good clustering. For 
the clustering criterion of DBI, theest scheme is essentially to minimize it as it is defined to choose the worst scenario of separation. Figure 1 presents the three criteria values for each $\mathrm{k}$ used in clustering.

The results indicate that no single number of $\mathrm{k}$ can optimize all the criteria. We chose to divide the data into six clusters, as all three criteria present a relatively good performance at $\mathrm{k}=6$, which is also appropriate to the content setting of five auction types.

The results of six clusters show specific participation patterns for online bidders, wherein layers in each cluster show their own auction type preferences. Figure 2 shows the corresponding details. Players in the first five clusters show bidding preferences for VP, GM, CDP, EDP, and FA auctions, respectively.

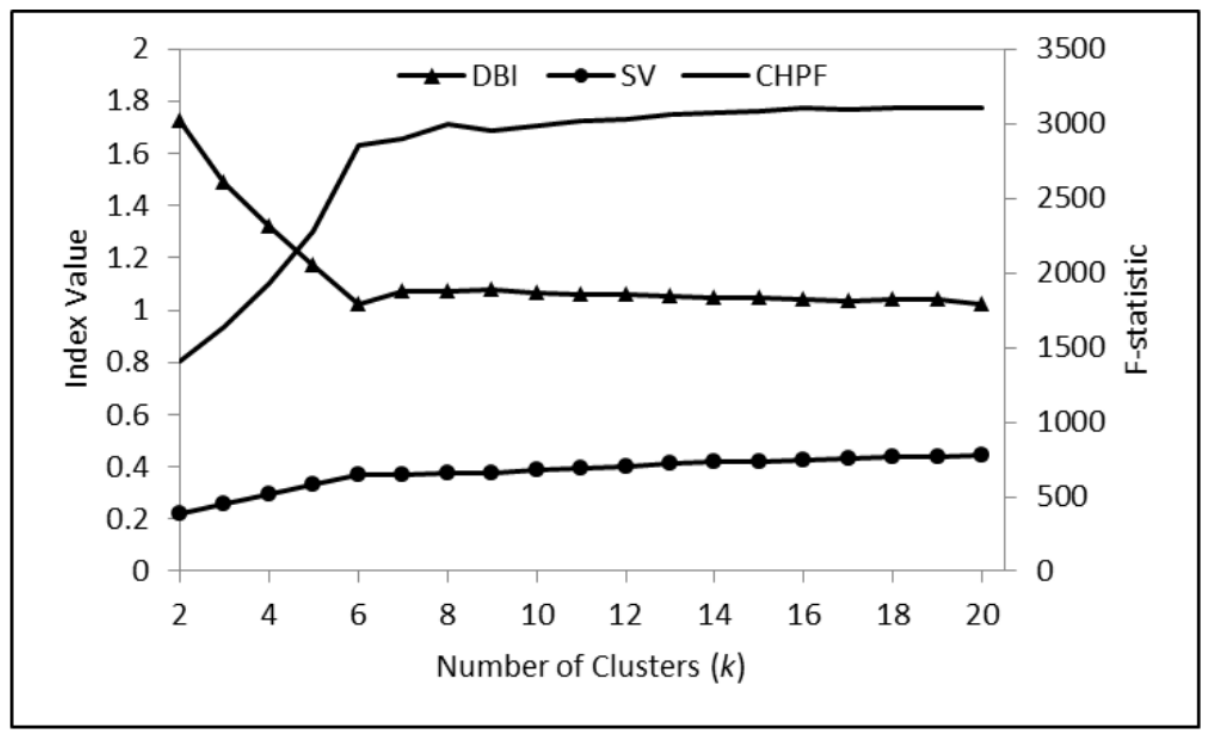

Figure 1. Mean scores for the CHPF, DBI and SV criteria k, respectively

Players paid most attention to only one type of auction and participated less in others. Players in Cluster 1 participated in more inVP auctions, which account for $70.7 \%$ of all auctions they have participated on average. The proportion relevant values of the other four clusters, cluster 2 to 5 , are $90.6 \%$ for GM, $90.4 \%$ for CDP, $89.1 \%$ for EDP, and 59.5\% Fairly.Players in Cluster 6 show no strong preference for a specific auction type. They devoted their biddings rather evenly to all the five types, although CDP has a relatively higher portion. Players that prefer FA auctions can be treated as risk-averse users; although they have already transferred from Free Member to Paid Members, they still preferred to participate in FA more. The cluster sizes for the 6 clusters are 1010, 401, 658, 308 , 1327 and 1946 respectively.

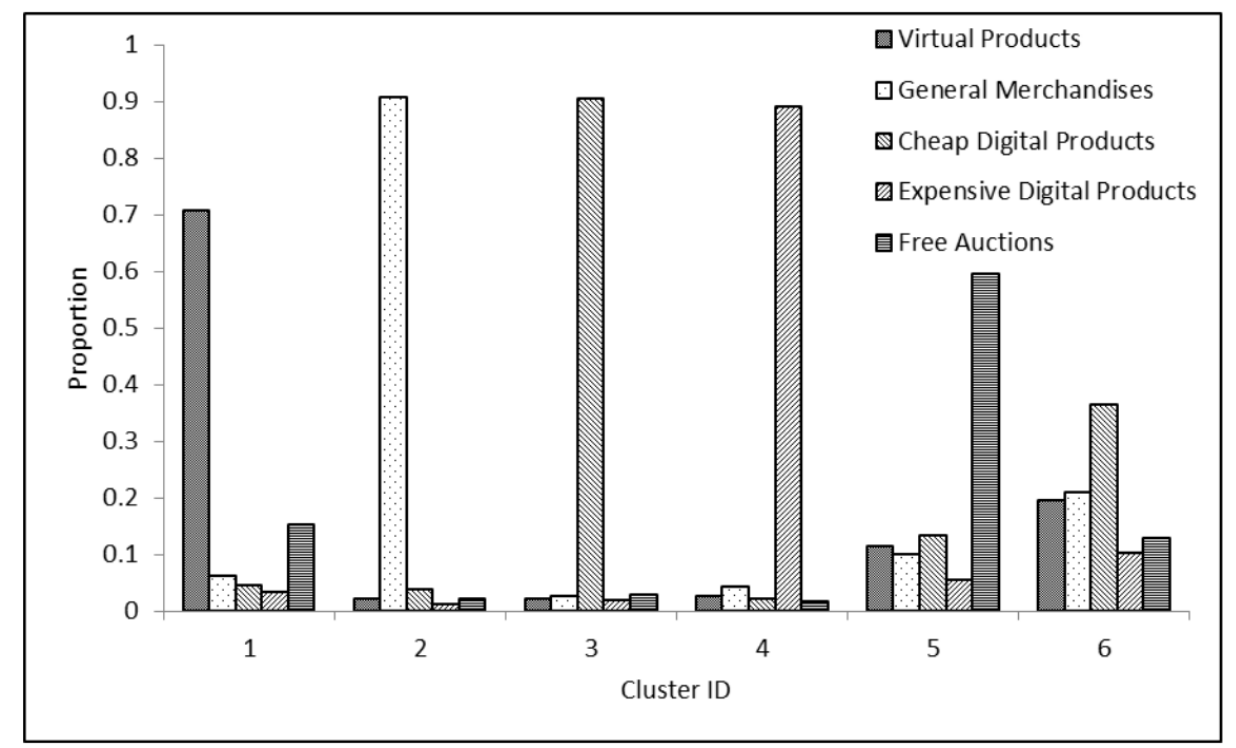

Figure 2. Proportion of participating auction numbers for different types in each cluster 
We have created a dummy variable called PreferenceTypeto indicatebidders' preferences. Here, auction type preferences can be identified according to the cluster each bidderbelongs. Different dummy variables named as TypeVP, TypeGM, TypeCDP, TypeEDP, TypeFA, and TypeALLare also created.

We did not use Num FreeAuction and NumPad Auction in our analysis as the Pearson correlation between the two variables and the variable Duration were significantly positive, with correlation of $r=0.537(p<0.01)$ andr $=0.671(p$ $<0.01$ ), respectively. The Results are consistent with the pay-per-bid auction mechanism that free auctions and bids were not available at any time in which the users were willing to participate. According to the rule set by the website, there are only two methods for players to obtain FBT for free auction participation (NumFreeAuction). The first is to persist in login to the website for a long time, which would induce a larger Duration. The other is the website rewards for the active participations in paid auctions, which is positively correlated with the number of paid auctions (NumPad Auction) .Hence, number of participations in free auctions (NumFreeAuction) is associated with or limited by Duration and NumPad Auction. The multicollinearity issues for AvgFreeAuction and Duration $(\mathrm{r}=-0.510, \mathrm{p}$ $<0.01$ ) were also taken into consideration when performing analyzes.

\section{Analysis Method and Results}

To test the hypotheses provided in Section 2, multiple linear regression models and regression models with beta distribution [26] were employed with different dependent variables. For testing the hypothesis H1, H2, and H6, the variable Web Profit that measured the profit contributed by players was used as the dependent variable to develop multiple linear regression models. The results are shown in Table 2.

Hypotheses $\mathrm{H} 3$ to $\mathrm{H} 5$ and $\mathrm{H} 7$ Test for the proportion of effective biddings, the dependent variable used was PropNoLossBid, which aims to measure the proportion of bidding that did not bring monetary loss to players. A regression model with beta distribution was used because the dependent variable is a continuous number ranging from zero to one.

From the sample of 5,650 players, there were eight observations with value 1 and 5,642 values were in the $(0,1)$ interval. For these eight players, they participated in and won only one paid auction, while all the others bids were free auctions. These eight observations were neglected as beta distribution requires the data bounded between 0 and 1 (excluding 0 and 1). The results are presented in Table 3.

Effects of PlayerAdherence: Results from Model 1 showed that both effects of Duration $(\beta=0.97$, s.e. $=0.10, p$ $<0.001)$ and AvgPaidAuction $(\beta=7.17$, s.e. $=1.11, \mathrm{p}<0.001)$ were positively related to WebProfit. Thus, $\mathrm{H} 1$ and $\mathrm{H} 2$ were supported.

Effects of Player Learning: Based on the results of Model 2in Table 3, the effect of time adherence, Duration $(\beta=$ 0.003 , s.e. $=0.001, \mathrm{p}<0.001$ ), was positively correlated with bidders' proportion of no loss bidding. It means that adhering to the website for a longer time can help players to improve the bidding ability for a higher proportion of effective biddings. Thus, H3 was supported. However, the negative impact of auction participation adherence, AvgPaidAuction $(\beta=-0.056$, s.e. $=0.009, \mathrm{p}<0.001)$, was observed on the proportion of no loss bidding. Therefore, H4 was not supported.

Effects of PlayerRisk Attitudes: TypeFA was used as a variable with value 1, denoting that players had free auctions preferences or risk-averse attitude. The results for the other clusters in Model 2 show a significantly negative effect relative to the cluster of TypeFA; it shows that players with monetary risk-averse attitude had a higher proportion of effective biddings. Thus, H5 was supported. 
Table 2. Result of multiple linear regression model

\begin{tabular}{|l|l|l|}
\hline & \multicolumn{2}{|c|}{ DV: WebProfit from bidders } \\
\hline Model 1 & Estimate & Standard Error \\
\hline Intercept & $-43.83 * * *$ & 5.49 \\
\hline RegTime & $0.60 * *$ & 0.21 \\
\hline TrickyName & $37.17 *$ & 14.85 \\
\hline TimeGap & -0.20 & 0.30 \\
\hline Duration & $0.97 * * *$ & 0.10 \\
\hline AvgPaidAuction & $7.17 * * *$ & 1.11 \\
\hline AvgPaidBid & $2.09 * * *$ & 0.07 \\
\hline TypeALL & 3.33 & 5.07 \\
\hline TypeVP & $16.76^{* *}$ & 5.59 \\
\hline TypeGM & 6.74 & 7.65 \\
\hline TypeCDP & 8.66 & 6.47 \\
\hline TypeEDP & 11.16 & 8.44 \\
\hline N & \multicolumn{2}{|c|}{5650} \\
\hline F test & \multicolumn{2}{|c|}{$09.1 * * *$} \\
\hline Multiple R2 & \multicolumn{2}{|c|}{} \\
\hline
\end{tabular}

Table 3. Results of the regression model with a beta distribution

\begin{tabular}{|l|l|l|}
\hline & \multicolumn{2}{|l|}{ DV: PropNoLossBid for bidders } \\
\hline Model 2 & Estimate & Standard Error \\
\hline Intercept & $-0.275^{* * *}$ & 0.039 \\
\hline RegTime & 0.000 & 0.002 \\
\hline TrickyName & $0.252 *$ & 0.100 \\
\hline TimeGap & -0.001 & 0.002 \\
\hline Duration & $0.003 * * *$ & 0.001 \\
\hline AvgPaidAuction & $-0.056^{* * *}$ & 0.009 \\
\hline AvgPaidBid & $-0.005^{* * *}$ & 0.001 \\
\hline TypeALL & $-1.196^{* * *}$ & 0.036 \\
\hline TypeVP & $-1.160^{* * *}$ & 0.040 \\
\hline TypeGM & $-1.358^{* * *}$ & 0.056 \\
\hline TypeCDP & $-1.365^{* * *}$ & 0.047 \\
\hline TypeEDP & $-1.443 * * *$ & 0.062 \\
\hline$\sigma=\alpha /(\alpha+\beta)$ & $-0.231 * * *$ & 0.014 \\
\hline $\mathrm{N}$ & \multicolumn{2}{|c|}{5642} \\
\hline Multiple R2 & \multicolumn{2}{|l}{0.2732} \\
\hline
\end{tabular}

Effects of PlayerPreference: For the player preference effects, Hypotheses 6 and 7 were mainly concerned on effects of having a specific auction type preference. The variable Type with value 1 denoted that players had no specific auction type preferences, while 0 means otherwise. In testing Hypothesis 6, dummy variables TypeEDP, TypeCDP, TypeGM, TypeAll, and TypeVP were used to perform the analysis. With preference of TypeFA as a base, the results in Model 1 show that the effects of all the other clusters are positive. While the absolute value for the cluster Type All is the smallest one, the results indicate that those who had no specific auction type preferences contribute less profit to the website, compared with players who have a specific auction type preference. Thus, H6 was supported. Model 2 was used to check the influence strength for each type of auction cluster. The effects for these dummy variables (with preference of TypeFA as a base) were all negatively related to the proportion of no loss bidding. The strengths of each preference group for the dependent variable, PropNoLossBid, can be sequenced according to the absolute value as follows: TypeEDP $(\beta=-1.443$, se $=0.062, \mathrm{p}<0.001)$, TypeCDP $(\beta=-1.365$, se $=0.047, \mathrm{p}<0.001)$, TypeGM $(\beta=$ -1.358 , se $=0.056, \mathrm{p}<0.001)$, TypeAll $(\beta=-1.196$, se $=0.036, \mathrm{p}<0.001)$, and TypeVP $(\beta=-1.160$, se $=0.040, \mathrm{p}$ $<0.001)$. The results showed that the strength for the effect of TypeEDP was the largest among all these five 
dummies, and as we mentioned, EDP auction was the type with the greatest monetary risk. Therefore, H7 was supported. The hypotheses testing results are summarized in Table 4.

Table 4. Hypotheses testing results

\begin{tabular}{|c|c|c|}
\hline Effects & Research Hypotheses & Results \\
\hline \multirow{2}{*}{$\begin{array}{l}\text { Player } \\
\text { Adhering }\end{array}$} & $\begin{array}{l}\text { H1: Players with longer lifetime contribute more profit to } \\
\text { entertainment shopping website. }\end{array}$ & Supported \\
\hline & $\begin{array}{l}\text { H2: Players who participate in auctions more frequently } \\
\text { contribute more profit to entertainment shopping website. }\end{array}$ & Supported \\
\hline \multirow{2}{*}{$\begin{array}{l}\text { Player } \\
\text { Learning }\end{array}$} & $\begin{array}{l}\text { H3: Players with longer lifetime make higher proportion of } \\
\text { effective biddings. }\end{array}$ & Supported \\
\hline & $\begin{array}{l}\text { H4: Players who participated in auctions more frequently make } \\
\text { higher proportion of effective biddings. }\end{array}$ & $\begin{array}{l}\text { Not } \\
\text { Supported }\end{array}$ \\
\hline $\begin{array}{l}\text { Player } \\
\text { Risk Attitude }\end{array}$ & $\begin{array}{l}\text { H5: Players with risk-averse attitude make a higher proportion } \\
\text { of effective biddings. }\end{array}$ & Supported \\
\hline \multirow{2}{*}{$\begin{array}{l}\text { Player } \\
\text { Preference }\end{array}$} & $\begin{array}{l}\text { H6: Players with a strong auction type preference contribute } \\
\text { more profit to the entertainment shopping website. }\end{array}$ & Supported \\
\hline & $\begin{array}{l}\text { H7: Players with a strong auction type preference with a larger } \\
\text { monetary risk make a lower proportion of effective biddings. }\end{array}$ & Supported \\
\hline
\end{tabular}

\section{Discussion}

Online entertainment shopping website integrates auction and entertainment factors together and operates to gain profit. Neglecting the operations of auction settings which can be easily observed and adjusted according to practice by the auctioneer, this study creates a data exploration and statistical method on how and how much the website can gain from online players with different characteristics and behaviors. The analysis is meaningful as online pay-per-biduction is a process of dynamic interactions between auctioneer and players, and a dynamic competition among all the unseen competitors. How they act to affect each other's behavior is a key issue in such conditions. From the auctioneer's view, they care about from whom they can gain or lose the revenue. From the players' perspectives, entertainment is one aspect they can capture and experience, but they value monetary benefits more.

First, because entertainment shopping websites are derived from conventional e-commerce websites, theories in traditional e-commerce should be considered to see whether there is a similar influence. Playeradherence was the first to be incorporated to measure a player's loyalty to the website. The multiple linear regression results showed that players who had a higher level of adherence in both dimensions of time and auction participations would contribute more profit to the website. However, players can only improve their effective bidding proportions through time dimension.

Second, considering that pay-per-auction is a special auction format, the monetary risk attitudes in traditional online auctions have also been examined. Risk-averse behaviors were shown by players who prefer TypeFin the models. The empirical results support that risk-averse players contributed relatively less profit to the website, which corresponds to common sense. Players with such attitude also generated a relatively higher proportion of no loss bidding with the accumulation of free biddings.

Third, except for these factors we are familiar with, the preference effect is the factor we focused on in this study. Cluster analysis results classified players into different groups according to their specific auction type preferences. These clusters worked as proxies for various types of preferences. Players preferring Type ALL are seen as having no specific auction type preferences. They lost less money in the websites than players who had specific auction type preferences. Compared to players with preferences, they also made more effective biddings with emotional influences, as the results showed a higher proportion of no loss biddings. For players that have a preference for TypeVP, they significantly lost more money on the website. However, the results showed that players with this type performed the best in the proportion of effective bidding among all other preference clusters. Products in this type have a low market value and the competition level is not that high. Players also did not devote too much sunk cost in this auction type. The results of regression with beta distribution also tell us clearly about the performance for effective biddings among different preference clusters. Players with auction type preferences significantly devote more loss biddings, indicating that the preferences should have a utility value for players. For players with different preferences, effects of preferences with different strengths can be observed. Players trapped in the preference cluster 
of TypeEDP showed the lowest proportion of no loss bidding, meaning that they bid more irrationally. Winning such an auction means a large amount of savings for the player; however, there is also a very high risk of losing money.

Finally, the website gains a large amount of profit from players in total through their bidding devotion. This is the reason why many players either quit from the website quickly (almost 50\% of players never participated in paid pay-per-bid auctions) or transfer into the preference cluster of TypeFA. Although some players gained a large amount of profit from the website, they only account for a very small proportion (around 8.2\%). At the same time, players can improve their bidding efficiency. Moreover, learning from the website for a longer time also helps players to perform better in bidding. The analysis for the players who obtained monetary benefits from the website also showed that the larger AvgPaidAutcion $(\beta=7.79, \mathrm{se}=3.95, \mathrm{p}<0.05)$ and AvgPaidBid $(\beta=4.18, \mathrm{se}=0.31, \mathrm{p}<0.001)$ are, the more monetary benefits can be obtained.

With the considerable amount of research works on pay-per-bid auctions, various theoretical methods or models have tried capturing the uncertainties that exist in pay-per-bid auctions. This study provides empirical results on the effects of factors from conventional e-commerce and online auctions and games. The effects of preferences for different auction types are also highlighted. Platt et al. [2] incorporated the risk preference into their theoretical model and achieved a better auction behavior explanation and ending price distributions prediction. Following this spirit, the impacts of the auction type preferences in auction bidding can also be considered incorporated into theoretical modeling work. Based on the empirical results, we suggest entertainment shopping business to make policies for the tradeoff between gaining profit and extending players' active lifetime. Preferences can be treated as another revenue source. Players in different preference clusters have different weights for revenue and entertainment, and here, preference value can be transferred into monetary value. Those who are good at bidding either through potential bidding skills or learning should be strategically limited, while those who are always losing should be focused on, in case of winning and losing polarizations.

There exist some limitations in this research. For example, it is believed that demographical data will have effects on users' behaviors, e.g., gender and age; however, we cannot test their effects due to data limitations. When we measure players 'adherence in time dimension, we use the duration between players' first and last participation in the website, but this is not accurate. In reality, we cannot observe players' login actions, which can be used to measure their active status accurately and reasonably. For future research directions, based on the results of this study, ways on how to quantify and evaluate the value of auction type preference would be incorporated into a unified model similarly with the spirit of [2], to better understand players' participation behaviors in such online entertainment shopping websites.

References

[1] A. Ghose and S. P. Han, A dynamic structural model of user learning on the mobile Internet. Working Paper. Leonard N. Stern School of Business, New York University. Available at http://people.stern.nyu.edu/aghose/research.html, 2011.

[2] B. C. Platt, J. Price and H. Tappen, The role of risk preferences in pay-to-bid auctions. Management Science, 59 (9), 2117-2134, 2013.

[3] C. I. Teng and W. W. Chen, Team participation and online gamers loyalty. Electronic Commerce Research and Applications, $13(1), 24-31,2014$.

[4] C. L. Hsu and H. P. Lu, Consumer behavior in online game communities: A motivational factor perspective. Computers in Human Behavior, 23 (3), 1642-1659, 2007.

[5] D. Choi and J. Kim, Why people continue to play online games: in search of critical design factors to increase customer loyalty to online contents. Cyber Psychology \& Behavior, 7, 11-24, 2004.

[6] D. Gefen, Trust and TAM in online shopping: an integrated model. MIS Quarterly, 27 (1), 51-90, 2003.

[7] D. L. Davies and D. W. Bouldin, A cluster separation measure. IEEE Transactions on Pattern Analysis and Machine Intelligence, PAMI-1 (2), 224-227, 1979.

[8] E. L. Deci and R. M. Ryan, Accessibility and stability of predictors in the theory of planned behavior. Journal of Personality and Social Psychology, 63 (5), 754-765, 1987.

[9] F. F. Reichheld and P. Schefter, E-loyalty. Harvard Business Review, 78, 105-113, 2000.

[10]F. Zhu and X. Zhang, Impact of online consumer reviews on sales: the moderating role of product and consumer characteristics. Journal of Marketing, 74 (2), 133-148, 2010. 
Jin Li \& Kwok Fai Tso / Vol. 2, No. 3, December 2019, pp. 102-112

[11] G. Ku, Auctions and auction fever: Explanations from competitivearousal and framing. Kellogg Journal of Organization Behavior, 1-32, 2000.

[12] G. Zacharia and P. Maes, Trust management through reputation mechanisms. Applied Artificial Intelligence, 14, 881-907, 2000.

[13]H. Lu and J. C. Lin, Predicting customer behavior in the market-space: a study of Rayport and Sviokla's framework. Information and Management, 40, 1-10, 2003.

[14] J. B. Walther and M. R. Parks, Cues filtered out, cues filtered in: computer-mediated communication and relationships. In Handbook of interpersonal communication (3rd ed., Pp. 529-563), Knapp, M. L., \& Daly, J. A. (Eds.), Thousand Oaks, CA, Sage, 2002.

[15] J. K. Murnighan, A very extreme case of the dollar auction. Journal of Management Education, 26, 56-69, 2002.

[16] J. W. Byers, M. Mitzenmacherand G. Zervas, Information asymmetries in pay-per-bidauctions: how Swoopo makes bank. The 11th ACM Conference on Electronic Commerce, Cambridge, 1-12, 2010.L. Escartin-Sorjonen, DealDash - Turning shopping into a game. The Huffington Post, June 14, 2012.

[17] M. T. P. Adam, J. Krämer, C. Jähnig, S. Seifert and C. Weinhardt, Understanding auction fever: a framework for emotional bidding. Electron Markets, 21 (3), 197-207, 2011.

[18] N. Augenblick, Consumer and producer behavior in the market for penny auctions: a theoretical and empirical analysis. Working Paper. Haas School of Business, University of California, Berkeley. Available at http://faculty.haas.berkeley.edu/ned/, 2011.

[19]P. A. Pavlou and D. Gefen, Building effective online marketplaces with institution-based trust. Information Systems Research, 15 (1), 37-59, 2002.

[20]P. J. Rousseeuw, Silhouettes: a graphical aid to the interpretation and validation of cluster analysis. Computational and Applied Mathematics, 20, 53-65, 1987.

[21] R. B. Calinski and J. Harabasz, J, A dendrite method for cluster analysis. Communication in Statistics, 3, 1-27, 1974.

[22] R. E. Anderson and S. S. Srinivasan, E-satisfaction and e-loyalty: a contingency framework. Psychology \& Marketing, 20, $123-138,2003$.

[23]R. Iyengar, Al. Ansari and S. Gupta, A model of consumer learning for service quality and usage. Journal of marketing research, XLIV, 529-544, 2007.

[24] R. J. Vellerand, Toward a hierarchical model of intrinsic and extrinsic motivation. Advance Experiment Social Psychology, 29, 271-360, 1997.

[25]R. Kieschnick and B. D. McCullough, Regression analysis of variates observed on (0,1): percentages, proportions and fractions. Statistical Modeling, 3, 193-213, 2003.S. Li, K. Srinivasan and B. Sun, Internet auction features as quality signals. Journal of Marketing, 73, 75-92, 2009.

[26] S. Narayanan and P. Manchanda, Heterogeneous learning and the targeting of marketing communication for new products Marketing Science, 28 (3), 424-441, 2009.

[27] S. S. Al-Gahtani and M. King, Attitudes, satisfaction and usage: factors contributing to each in the acceptance of information technology. Behavior and Information Technology, 18 (4), 277-297, 1999.

[28] T. Erdem and M. P. Keane, Decision-making under uncertainty: Capturing dynamic brand choice processes in turbulent consumer goods market. Marketing Science, 15 (1), 1-20, 1996.

[29] T. Erdem, M. P. Keane and B. Sun, B, A dynamic model of brand choice when price and advertising signal product quality. Marketing Science, 27 (6), 1111-1125, 2008.

[30] T. Hinnosaar, Penny auctions are unpredictable. Working Paper. Department of Economics, Northwestern University. Available at http://toomas.hinnosaar.net/, 2010.

[31] Y. Chen and J. Xie, Third-party product review and firm marketing strategy. Marketing Science, 24 (2), $218-240,2005$.

[32] Y. Huang, P. V. Singh and K. Srinivasan, Crowdsourcing new product ideas under consumer learning. Management Science, 60 (9), 2138-2159, 2014.

[33] Y. Zhao, S. Yang, V. Narayan and Y. Zhao, Modeling consumer learning from online product reviews. Marketing Science, 32 (1), 153-169, 2013. 